\title{
Necessidades de adolescentes com sofrimento psíquico
}

\section{Necessities of adolescents with mental disorders}

\author{
Elisabete Ferreira Mângia ${ }^{(1)}$, Cecília Neves Assumpção ${ }^{(2)}$, \\ Jacqueline Mendonça Quinta ${ }^{(3)}$, Maria de Fátima Rufino ${ }^{(4)}$
}

\begin{abstract}
MÂNGIA, E. F.; ASSUMPÇÃO, C. N.; QUINTA, J. M.; RUFINO, M. F. Necessidades de adolescentes com sofrimento psíquico. Rev. Ter. Ocup. Univ. São Paulo, v. 14, n. 3, p. 123-32, set./dez. 2003.
\end{abstract}

RESUMO: O presente estudo tem como finalidade identificar as necessidades dos adolescentes com transtorno mental, objetivando a formulação de ações mais efetivas que possam orientar a prática dos serviços destinados a esta população. Para tanto, realizou uma pesquisa exploratória que buscou discutir e relacionar o referencial teórico com os "perfis ideais", construídos hipoteticamente a partir das experiências do grupo que realizou este trabalho. $\mathrm{O}$ estudo das necessidades possibilitou identificar os atores que devem estar envolvidos na resposta aos problemas de saúde apresentados, subsidiar a análise e planejamento das ações de prevenção e atenção em saúde mental, sugerindo alternativas no campo da instituição e das políticas de saúde.

DESCRITORES: Psiquiatria geriátrica. Terapia pela arte.

\section{INTRODUÇÃO}

$\mathrm{D}$ iscutir saúde mental na adolescência provoca muitas dúvidas e diferentes interpretações. Temos que discriminar o que é próprio desta fase da vida dos transtornos que podem ter início neste período. Dependendo do olhar do observador constatamos que as interpretações sobre o que se considera transtorno mental são controversas. Identificamos também, que os valores culturais, implicam em diferentes atribuições de significados, opções de convivência e formas de abordagem e tratamento.

Para a Organização Mundial da Saúde, os

\footnotetext{
(1) $\operatorname{Prof}^{\mathrm{a}} \mathrm{Dr}^{\mathrm{a}}$ do Departamento de Fisioterapia, Fonoaudiologia e Terapia Ocupacional da FMUSP.

(2) Psiquiatra, Psicodramatista, Coordenadora do Centro de Atenção Psicossocial para Adolescentes de Vila Prudente. e-mail: ceciliaassumpcao@uol.com.br

(3) Fonoaudióloga, Auxiliar de Coordenação do Centro de Atenção Psicossocial para Adolescentes de Vila Prudente. e-mail : jacquinta@ilg.com.br

(4) Psicóloga, Psicodramatista, Assessora de Saúde Mental da Coordenadoria de Saúde da Penha. e-mail: mfrufino@ig.com.br Endereço para correspondência: Rua Cipotânea, 51, Cidade Universitária, São Paulo, CEP 05360-160, e-mail: centroto@edu.usp.br.
} 
transtornos mentais e comportamentais devem ser entendidos como:

\begin{abstract}
“(...) condições clinicamente significativas caracterizadas por alterações do modo de pensar e do humor (emoções) ou por comportamentos associados com angústia pessoal e/ou deterioração do funcionamento. Os transtornos mentais e comportamentais não constituem apenas variações dentro da escala do 'normal' sendo antes fenômenos claramente anormais ou patológicos" (OMS/OPAS, 2001, p.47).
\end{abstract}

Neste trabalho, optamos pela utilização da terminologia "transtornos severos e persistentes" para definir tanto os quadros de psicose com início na infância, quanto os quadros de psicose com início na adolescência. Incluímos também a categorização "transtorno mental na adolescência" para os transtornos de humor e os quadros de ansiedade. Consideramos ainda, os casos de risco psicossocial que podem ou não apresentar um transtorno associado.

A identificação das necessidades dos adolescentes com sofrimento psíquico é uma das tarefas mais importantes que os serviços em saúde mental para adolescentes devem discutir e investigar.

Nos últimos cinqüenta anos, o movimento em favor dos direitos humanos, o desenvolvimento de novas formas de intervenção psicossocial, bem como a incorporação por parte da Organização Mundial da Saúde de componentes sociais e mentais na definição de saúde, definiram mudanças significativas nos projetos assistenciais e desencadearam os processos de reformas psiquiátricas em países da Europa e nos EUA, caracterizadas pela crítica ao modelo centrado no asilamento psiquiátrico e pela promoção da assistência centrada na comunidade, que repercutiram em todo o mundo (AMARANTE, 1997; BARROS, 1994; OMS/ OPAS, 2001).

No Brasil, o processo da reforma psiquiátrica apresenta ainda poucos avanços em relação à atenção de adolescentes com sofrimento psíquico, embora os estudos epidemiológicos mundiais e nacionais apontem para a relevância do problema. Deve-se levar em conta também que, em nosso país, crianças e adolescentes constituem $40 \%$ da população e faltam diretrizes em relação a cuidados de saúde e, mais especificamente, de saúde mental para estas faixas etárias. Dados epidemiológicos indicam a prevalência de 10 a $15 \%$ de transtornos mentais entre crianças e adolescentes, chegando a $21 \%$ se tomarmos a população de adolescentes com mais de 15 anos. O início de diversos transtornos mentais é muito mais precoce do que se julgava, há aumento das taxas de suicídio entre os jovens em diversas regiões do mundo. Além disso, 50\% desses transtornos tendem a produzir incapacidades permanentes (SAGESSE; COSTA LEITE, 1999, p.197).

Pesquisa realizada pela Universidade de São Paulo em conjunto com a Universidade de Londres, estimou que cerca de 3,4 milhões de crianças e adolescentes brasileiros, entre 7 e 14 anos, sofrem de transtornos psiquiátricos e que há insuficiência de profissionais envolvidos na atenção em saúde mental para essa população (ALMEIDA, 2003, p.30).

Almeida Filho, estudando uma amostra de 829 crianças de 5 a 14 anos residentes em Salvador - Bahia, estimou em 23,2\% a prevalência global de transtornos mentais (ALMEIDA FILHO, 1982 apud LAURIDSEN, 1998, p.39).

Em 2001, foi avaliado que, nos Estados Unidos, um em cada dez jovens apresentava transtorno mental e que menos de um em cinco recebia alguma atenção. 'É muito provável que a situação seja ainda menos satisfatória em grandes regiões do mundo em desenvolvimento(...)" (OMS/OPAS, 2001, p. 64), como no caso do Brasil. Cifras de prevalência mundiais variam consideravelmente apontando dados de que 10 a $20 \%$ das crianças possuem um ou mais transtornos mentais.

As necessidades de adolescentes com transtorno mental e daqueles que, embora não apresentem transtorno clínico definido, são encaminhados em número elevado aos serviços, por escolas e por outras instituições, exigem o desenvolvimento de um novo olhar e de uma nova escuta, na perspectiva de planejar e desenvolver ações de atenção em saúde mental singulares e diferenciadas.

Os serviços públicos, de atenção primária ou especializados, que oferecem atenção em saúde mental para adolescentes devem se apropriar da compreensão das necessidades de saúde dessa população para planejarem e organizarem o seu trabalho. Questões como a alta incidência de transtornos mentais nos adolescentes; a baixa resolutividade dos atendimentos e a ausência de identificação precisa das necessidades de saúde, devem ser consideradas, para possibilitar a discussão e elaboração de novas estratégias de intervenção e prevenção.

Nessa direção, o estudo das necessidades pode ser o ponto de partida pois permite que a queixa, freqüentemente trazida pela demanda mais imediata, possa ser deslocada para a busca do conhecimento mais complexo do problema, para a identificação do contexto e dos atores envolvidos na produção do sofrimento psíquico, para a avaliação da gravidade e repercussões trazidas pelo problema e para a identificação dos recursos para enfrentá-lo. 
A adolescência pode ser caracterizada como "um período da vida em que há importantes transformações físicas e mentais articuladas, em nossas sociedades, a um amplo redimensionamento de identidades e papéis sociais" (AYRES; FRANÇA JUNIOR, 1996). É um período de desenvolvimento e de construção de projetos, portanto a intervenção no curso dos transtornos mentais nesta fase pode ser decisiva e de fundamental importância, para a vida adulta.

Este trabalho buscou identificar as necessidades dos adolescentes com transtornos mentais; identificar quais são as necessidades dos adolescentes que embora não apresentando transtornos clínicos definidos necessitam de algum nível de atenção em saúde mental; apreender como a equipe do serviço estudado identifica as necessidades dos usuários e relacionar as necessidades identificadas ao planejamento da atenção em saúde mental para adolescentes com sofrimento psíquico.

\section{CONFIGURAÇÃO METODOLÓGICA}

Este estudo configurou-se enquanto uma pesquisa exploratória que buscou, a partir da reflexão sobre as características da clientela usuária de um serviço público de saúde mental, apreender e identificar as necessidades em saúde mental de adolescentes com transtorno mental, tendo em vista a formulação de ações mais efetivas que pudessem orientar a prática dos serviços destinados a esta população. Dada a abrangência e complexidade desse objetivo, se inscreve no campo da investigação qualitativa (MINAYO, 1997; VASCONCELOS, 2002).

O trabalho foi efetivado por meio de pesquisa bibliográfica e documental e pelo processo de observação de campo e realização de grupo de consenso a partir da experiência vivida pelas autoras no Centro de Atenção Psicossocial (CAPS) para adolescentes de Vila Prudente - situado na zona leste do município de São Paulo. Da experiência prática foram construídos "perfis-ideais" da clientela atendida pelo serviço e das práticas desenvolvidas que apoiaram a reflexão e questionamento sobre as necessidades de saúde mental dos adolescentes e as possibilidades de respostas desenvolvidas pelos serviços. Os "perfis-ideais" foram elaborados a partir da identificação de características comuns, tais como: diagnóstico, queixas, histórias de vida, situação familiar e outros.

O trabalho buscou ancoragem teórica em autores que tem se preocupado em definir o conceito de necessidades de saúde a partir da demanda e da oferta de serviço, especialmente Cecílio (2001) e de Schraiber e Mendes-Gonçalves (1996).

Encontrou também subsídios em autores que discutem as características dos novos serviços de saúde mental no contexto da reforma psiquiátrica, especialmente Saraceno (1909), Kinoshita (1996) e o Relatório de Saúde para o Mundo 2001 (OMS/OPAS 2001).

A elaboração da discussão consistiu em correlacionar o referencial teórico com os "perfis- ideais" formulando concepções quanto ao modelo assistencial e ações relevantes que possam produzir respostas efetivas às necessidades da população.

\section{IDENTIFICAÇÃODOCONTEXTODAPESQUISA}

OCAPS para Adolescente de Vila Prudente tem como missão oferecer atenção intensiva, semi-intensiva e não intensiva aos usuários com transtornos mentais que tem início na infância ou adolescência. São objetivos desse serviço: 1. evitar a primeira internação e reinternações; 2 . promover atenção em saúde mental sem exclusão do usuário do convívio social e familiar, possibilitando a manutenção de atividades rotineiras - escola, atividades sociais, esportivas e de trabalho; 3 . promover condições para o desenvolvimento compatível com as possibilidades de cada usuário; 4. melhorar a comunicação entre os membros da família; 5 . melhorar as interações sociais da família na comunidade; 6. promover autonomia e independência, por meio de ações que estimulem desde o cumprimento de pequenas tarefas, de cuidados pessoais e do cotidiano, como também realizações para o próprio projeto de vida.

OCAPS recebe e oferece acolhimento para todos os adolescentes que chegam ao serviço, desde que sejam moradores da área de abrangência do serviço e tenham de 12 a 18 anos. Na maioria das vezes o primeiro contato é telefônico, realizado por um técnico. São investigadas a queixa principal, a idade, a localidade da moradia e a natureza do encaminhamento.

É realizada a primeira entrevista com o adolescente e a família na qual se avalia a dinâmica familiar, inserção social e escolar, e se tenta elaborar uma hipótese diagnóstica.

Ao final desse processo o caso é levado para discussão com toda a equipe para a elaboração e proposição de um projeto terapêutico.

O serviço oferece atendimento individual, oficinas terapêuticas, grupos terapêuticos, atendimento às famílias, visitas domiciliares, eventos e atividades externas. A inserção na escola é feita por meio de discussões sistemáticas entre as equipes da saúde e da educação. 


\section{QUEM A UNIDADE ATENDE}

1) O CAPS recebe usuários com transtornos mentais severos com início na primeira infância e na adolescência, muitas vezes expostos a situações de risco, como suicídio, uso de drogas, comportamentos violentos e internação desnecessárias.

2) Adolescentes que apresentam sintomatologia intensa e duradoura, compatível com quadros de ansiedade e depressão. São freqüentes casos com sintomatologia de pânico, quadros obsessivocompulsivos, ansiedade, quadros conversivos e com sintomatologia psicossomática.

3) Adolescentes em risco psicossocial que chegam ao CAPS quando há suspeita de alguma intercorrência psiquiátrica/psicológica associada. Estão neste grupo, meninos de rua e adolescentes vindos de abrigos, órfãos ou abandonados, com a queixa de comportamentos inadequados e falta de adaptação. Ainda neste grupo localizamos adolescentes encaminhados pelas escolas, com "dificuldade de aprendizagem" e "mau comportamento".

\section{APONTAMENTOS SOBRE AS NECESSIDADES DE SAÚDE}

Definir e discutir necessidades de saúde é uma tarefa complexa, Cecílio (2001) classifica as necessidades de saúde em quatro categorias.

a) A primeira diz respeito às boas condições de vida, relacionada a fatores ambientais e externos ao indivíduo, que determinam o processo saúde-doença, considerando o modo de produção no qual o sujeito está inserido.

b) A segunda categoria diz respeito à possibilidade de acesso e consumo da assistência em saúde capaz de melhorar e prolongar a vida.

c) A terceira diz respeito à criação de vínculos efetivos e afetivos entre usuário e equipe e/ou profissional de saúde. Não se refere apenas ao simples pertencimento do usuário a uma determinada unidade de saúde, mas sim a possibilidade de estabelecer uma relação na qual usuário e profissional, no encontro de suas subjetividades, possam enfrentar os problemas de saúde.

d) $\mathrm{O}$ quarto e último conjunto diz respeito à necessidade do sujeito de ampliar o grau de autonomia em seu "modo de levar a vida". Refere-se também, à necessidade de educação e informação em saúde, que incrementa a possibilidade do sujeito reconstruir sua vida e lutar pela satisfação de suas necessidades.

As necessidades de saúde estão associadas à assistência, na medida em que a necessidade é traduzida pela procura do serviço de saúde a partir da identificação de uma alteração física, orgânica ou de um sofrimento ainda não identificado fisicamente, ou algo que o sujeito reconhece como uma falta. $\mathrm{O}$ usuário busca pela solução do problema, e lança mão do conhecimento baseado em sua experiência, de demandas tratadas anteriormente ou na experiência de outros usuários que levaram suas demandas para o sistema de saúde. Por outro lado, a oferta de um determinado serviço estabelece a difusão de um conhecimento sobre a resposta que um problema de saúde pode ter. Dessa forma,

“...o modo de organizar socialmente as ações em saúde para a produção e distribuição efetiva dos serviços será não apenas a resposta a necessidades, mas imediatamente, contexto instaurador de necessidades. Assim, há uma conexão circular entre a organização da produção, a oferta ou distribuição de serviços e o seu consumo" (SCHRAIBER; MENDES-GONÇALVES, 1996, p.30).

Assumir a existência dessa circularidade, coloca para os profissionais a tarefa de, a partir do ponto de vista do usuário e de suas experiências, descobrir novas necessidades não codificadas apriorísticamente pelos serviços.
"Nas sociedades modernas, as necessidades em saúde, assim com outras, tem tanto um valor de uso como um valor de troca. Elas se materializam em produtos que podem ser utilizados e comercializados, através do mercado. Por esta razão, elas são manipuladas socialmente, segundo os interesses que predominam. De acordo com interesses, parâmetros e regras instauradas no mundo capitalista, ordenados por sua lógica de consumo-lucro crescente, certas necessidades são criadas e outras ocultadas. Desse modo, as respostas dos serviços às necessidades são contextos "instauradores de necessidades"' (SCHRAIBER; MENDES-GONÇALVES, 1996, p. 30).

Devemos considerar também que as necessidades de saúde não são homogêneas e na sua identificação deve-se levar em conta as desigualdades sociais, o acesso aos recursos, o modo de vida e, no caso da saúde mental, fatores singulares determinantes da história de vida de cada sujeito e de seu grupo familiar.

"Carências e necessidades devem ser consideradas e reconhecidas igualmente. Traduzindo isto para o campo da saúde, significa dizer que são legítimas as reivindicações da clientela feitas aos serviço de saúde, ainda que elas representem uma certa alienação das necessidades essenciais. Nesse campo, é preciso valorizar as carências e a sua satisfação, traduzidas em demandas sociais, pois somente assim é possível engajar pessoas em processo de autodeterminação. E esta, enquanto uma necessidade 
essencial, não pode ficar relegada a segundo plano" (MANDU; ALMEIDA, 1999, p.59).

Observamos, na experiência dos serviços de saúde mental, que a demanda trazida pelas famílias dos usuários se reduz, muitas vezes na procura de um médico psiquiatra e fornecimento de medicamentos. Consideramos que esta demanda trazida imediatamente junto com a queixa é o meio pelo qual podemos iniciar a relação com o usuário. A tarefa é compreender o que pode estar contido nesta queixa e poder identificar em parceria com a família e o adolescente, as necessidades de saúde e os cuidados a serem desenvolvidos.

\section{IDENTIFICANDO OS GRUPOS ATENDIDOS E PROPONDO AÇÕES}

Com o intuito de reconhecer quais as necessidades dos adolescentes com sofrimento psíquico, e definir ações compatíveis a essas necessidades e considerando que o critério diagnóstico, freqüentemente utilizado pelos serviços, opera como um elemento simplificador da condição real de cada sujeito e do complexo processo de produção e emergência daquilo que se convenciona reconhecer como sintoma, a reflexão operada nesse trabalho buscou reconhecer e descobrir, a partir de "perfis-ideais" reconhecidos pelo olhar técnico, ao menos parcialmente, a complexidade do objeto encoberto pela abstração diagnóstica. Dessa forma, a partir da linguagem diagnóstica buscou-se apreender a diversidade das histórias, características sócio-familiares, queixas, situações e necessidades a serem enfrentadas pelos serviços.

No primeiro grupo estão os adolescentes com transtornos mentais severos, reconhecidos pelo olhar diagnóstico como psicoses, distribuídos em dois subgrupos: a) processos com início na infância e b) processos com início na adolescência. No segundo grupo estão os casos considerados de risco psicossocial. Buscou-se agrupar e discutir as características nos seguintes eixos: fragilidade das respostas institucionais, respostas à família e rede social, escolaridade, autonomia e independência

\section{OS TRANSTORNOS MENTAIS SEVEROS COM INÍCIONA INFÂNCIA}

Reconhecemos neste grupo adolescentes que apresentaram prejuízos importantes na interação social, linguagem, comunicação e comportamento ao longo da infância. Freqüentemente são histórias que tem início no primeiro e segundo anos de vida, com trajetórias muito singulares. Muitas famílias trazem relatos sobre a passagem por inúmeros serviços de psiquiatria e psicologia. Outras estão procurando o serviço especializado em saúde mental pela primeira vez, nestes casos freqüentemente se observa níveis altos de isolamento social do adolescente e da própria família.

As famílias apresentam diferentes pedidos: relatórios e laudos vinculados à obtenção de benefícios; tratamento para os sintomas mais expressivos (agressividade, falta de sono, recusa à alimentação); busca de internação em instituição asilar.

Observa-se o cansaço, desânimo e descrença dos pais em relação às respostas possíveis e histórias sobre a recusa de atendimento por muitos serviços de saúde.

Alguns adolescentes apresentam história de institucionalização de meses ou anos e hábitos desenvolvidos nos processos de institucionalização.

\section{FRAGILIDADEDAS RESPOSTASINSTITUCIONAIS}

Constatamos que, apesar de terem sido submetidos a diversos tratamentos ao longo dos anos, há muita descontinuidade e ausência de respostas efetivas em termos da construção de projetos terapêuticos e de vida. A resposta mais freqüente é a medicamentosa, privilegiando o atendimento psiquiátrico e neurológico.

Esta experiência é incorporada pela família e pelo adolescente, de forma a gerar desesperança, perda das expectativas e ausência de protagonismos.

Dentre os adolescentes que chegam ao serviço sem tratamento anterior, nota-se grande isolamento e prejuízos importantes no desenvolvimento, nesses casos é importante refletir sobre as necessidades de socialização. Observam-se comportamentos em desacordo com padrões sociais cotidianos (comer com as mãos, falta de hábitos básicos de higiene e cuidados pessoais, etc) que mobilizam as equipes e resultam nos primeiros cuidados propostos.

\section{RESPOSTAS À FAMÍLIA E REDE SOCIAL}

Observa-se que as necessidades explicitadas pela família na queixa inicial são muito limitadas e encobrem questões mais complexas. Dentre aquelas famílias que tem um longo histórico em serviços psiquiátricos e de psicologia, constatamos a incorporação desses modelos de atendimento que passam a ser referência para a identificação das necessidades e atribuição de valor, por exemplo a consulta médica, o remédio e a escuta psicológica. É importante que se possa revelar para os usuários e 
suas famílias a concepção do tratamento, embasada em ações multidisciplinares integradas no contexto de um projeto terapêutico e institucional complexo.

Percebe-se a necessidade da ampliação da escuta e do diálogo permanente com as famílias no sentido de que possam reconhecer as suas necessidades reais, apropriar-se da construção de projetos mais complexos e desenvolver novos repertórios relacionais que permitam um desempenho mais ativo no cuidado ao adolescente.

Constata-se que o atendimento das famílias pode proporcionar o suporte necessário para que possam lidar com os transtornos severos e persistentes, sem ficarem extremamente identificadas com o processo ou dele se afastarem demais.

Reconhece-se também a importância do estabelecimento de um contrato claro que formalize os passos da escuta, a busca da identificação das necessidades do adolescente e da família, e desenhe metas e compromissos a serem desenvolvidos pelo serviço e pela própria família.

\section{ESCOLARIDADE}

Para esse grupo os processos educacionais são muito limitados, mesmo quando se trata de adolescentes que não apresentam déficit intelectual ou outros prejuízos que alteram o desenvolvimento normal. Alguns freqüentam escolas especiais, que não atendem às suas necessidades e poucos estão integrados a escola normal, onde a inclusão ainda é uma perspectiva para o futuro pois faltam estratégias efetivas, que garantam o direito ao acesso à educação. A grande maioria, nunca ou muito pouco, freqüentou uma instituição de ensino, de qualquer tipo.

A falta de acesso à educação, para aqueles cujo acometimento tem início na infância acarreta prejuízos secundários, como a defasagem na aprendizagem e a falta de oportunidades de interação que a escola poderia propiciar.

\section{Autonomia e independência}

Nesse grupo se observam prejuízos no desenvolvimento físico e psíquico. São jovens que necessitam de acompanhantes para chegarem ao serviço, não possuem autonomia para realizar tarefas como higiene e alimentação. Encontram-se graus variados de déficit de aprendizagem escolar, interesses restritos a algumas áreas específicas; alguns, embora alfabetizados, não apresentam interesse pelo conteúdo ou pelas possibilidades de escolha de leituras. Geralmente não apresentam interesses próprios, não fazem pedidos por mudanças ou novidades, e quando demonstram interesse, persistem no mesmo tema ou na mesma atividade. Em alguns casos, se pode observar sinais de angústia e inquietação permanentes.

São adolescentes que possuem uma rotina extremamente empobrecida, ficam muito tempo em casa e poucos freqüentam a escola ou outro equipamento social. Alguns desses usuários parecem não ter consciência de sua idade, e outros ao se perceberem como adolescentes vivenciam um estado maior de sofrimento vinculado ao fato de se sentirem diferentes.

\section{na adolescência}

Os transtornos mentais severos com início

O que caracteriza esse grupo é a presença de mudanças abruptas e intensas nos comportamentos, hábitos e pensamento. Aparecimento de pensamento desorganizado, delirante, acelerado ou isolamento, mutismo e embotamento.

Chegam trazidos pela família que apresenta surpresa e aflição em relação às mudanças dos seus filhos. Em muitos casos as famílias já tomaram contato com algum diagnóstico atribuído numa primeira consulta médica, especialmente os de esquizofrenia e transtorno bipolar, que costumam causar efeitos muito negativos sobre as famílias.

No geral, são relatadas histórias de vida sem indícios de transtorno mental e escolaridade adequada. Freqüentemente há referências à sociabilidade restrita, timidez e isolamento. Algumas vezes encontra-se referências à pouca adaptabilidade em períodos de crise e conflito.

\section{Fragilidade das respostas institucionais}

Na avaliação das situações relatadas se observa que o aparecimento e instalação abrupta dos sintomas, determinam a busca de serviços de emergência e/ou ambulatoriais. Freqüentemente esses adolescentes recebem diagnósticos psiquiátricos e medicação a partir de uma única observação. Algumas vezes, foram submetidos à internação psiquiátrica desnecessária e estigmatizante. Constata-se que a precipitação na atribuição de um diagnóstico ou na decisão da internação não é acompanhada de medidas que assegurem a continuidade do cuidado, nem tampouco a definição clara de um projeto terapêutico efetivo.

Em alguns casos se observa que após a primeira crise, permanecem por um período em casa sem que as famílias procurem por tratamento em saúde. 
Muitas famílias buscam primeiramente soluções no âmbito das práticas religiosas ou espirituais.

\section{Respostas à família e rede social}

Nestes casos observamos que a queixa inicial e a demanda da família é pela abolição dos sintomas para que o adolescente retome sua rotina habitual. A necessidade de atenção em saúde nestes casos se assemelha às do grupo relatado anteriormente, ou seja, oferecer para a família o espaço para a escuta e compreensão da situação e suporte para o período de crise.

\section{Escolaridade}

No geral observa-se que, em sua maioria, esse grupo apresenta escolaridade compatível com a idade. São afastados da escola após a instalação da primeira crise. Identifica-se a necessidade de possibilitar e garantir que a interrupção da vida escolar seja minimizada e que se possa compartilhar com a escola esse momento, evitando a discriminação e criando medidas protetivas.

\section{Autonomia e independência}

$\mathrm{Na}$ maior parte das situações se observa a perda temporária da capacidade de autonomia, discernimento, crítica e decisão. Após o episódio agudo há a necessidade de que se avalie as consequiências desse evento para que se busque estratégias de recuperação da autonomia e independência.

\section{As situações de risco psicossocial}

Consideramos neste grupo os adolescentes: moradores de rua ou que permanecem a maior parte do seu tempo nas ruas, moradores de abrigo, separados de suas famílias ou órfãos, aqueles que se encontram em situação de confronto, exclusão ou discriminação no ambiente escolar. Além disso em parte deste grupo, está presente também algum grau de confronto com a lei.

Chegam ao serviço por intermédio da instituição responsável por eles ou pela escola. A queixa mais frequiente diz respeito às manifestações de agressividade, usualmente descrita como desproporcional e inadequada aos eventos que a desencadearam, agitação e desobediência. No caso dos abrigos, os cuidadores, consideram impossível administrar tais situações, que são atribuídas a um suposto quadro psiquiátrico.

É um grupo heterogêneo e de difícil caracterização. Verifica-se que apenas pequena parcela dos casos encaminhados ao serviço apresenta algum transtorno mental.

Nas histórias de vida apresentam em comum as situações de abandono, a ausência de vínculos duradouros, desestruturação familiar, violência por parte dos familiares ou das instituições que os abrigaram. Há também casos de orfandade.

\section{Fragilidade das respostas institucionais}

Primeiramente, consideramos que essa clientela necessita de um espaço que a proteja de mais um risco, o da institucionalização em saúde mental. Os educadores e as equipes dos abrigos, consideram que as alterações de comportamento e dificuldades de relacionamento são um problema de saúde mental, localizado no adolescente para o qual se busca o cuidado.

Há situações evidentes de sofrimento psíquico, presentes nos dois lados: o trabalhador não consegue cumprir sua tarefa de cuidar ou educar, e o adolescente não forma vínculo, sente-se ameaçado ou abandonado pelos cuidadores ou pela escola. Tais situações deveriam ser administradas conjuntamente, por todos os envolvidos no cuidado ou na educação ao adolescente e não focalizadas apenas nas queixas comportamentais.

Observamos que crianças e adolescentes abandonados e sem vínculos afetivos duradouros, apresentam pouca tolerância a frustrações e limites, assim como dificuldades para lidar com os momentos críticos, em decorrência de sua própria trajetória de vida e institucionalização.

Os casos mais críticos, quando há algum transtorno mental associado, devem receber atenção em saúde mental que os proteja dos processos de internação.

Torna-se evidente a necessidade de um trabalho intersetorial especialmente com as instituições que oferecem abrigo. Os trabalhadores dos abrigos, encontram-se pouco instrumentalizados para lidarem com as crianças e adolescentes abandonados e com o impacto emocional trazido por suas histórias de vida.

As famílias desestruturadas também se encontram numa situação de risco e não conseguem oferecer um modelo organizador para a vida psíquica de seus componentes. Constata-se que esse grupo tem pouco acesso aos serviços de saúde e de saúde mental. 


\section{Respostas à família e rede social}

Nos casos de abandono ou de pais considerados inaptos para exercerem suas funções, o Estado delega para instituições como lares e abrigos a responsabilidade de exercerem o papel originariamente atribuído à família. A equipe técnica dos abrigos é responsável pelas funções de cuidar, proteger, alimentar, dar afeto e promover o desenvolvimento, e reproduz as queixas dos pais e da família.

Assim como nas famílias identificamos dinâmicas mais ou menos favoráveis ao desenvolvimento dos adolescentes, é necessário que se considere que o mesmo ocorre em relação à dinâmica institucional dos abrigos. Consideramos a necessidade de oferecer atenção em saúde mental para os profissionais dos abrigos, na mesma lógica que se atende às famílias.

Para responder de forma satisfatória às necessidades dos adolescentes em risco psicossocial, é necessário articular uma rede de apoio, integrada pelos serviços de saúde, educação, Conselho Tutelar, abrigos, etc, de forma a propiciar ações que respondam as múltiplas necessidades dos adolescentes.

\section{Escolaridade}

A inserção escolar desse grupo está fora dos padrões socialmente esperados Há a presença de baixo rendimento escolar, apesar da capacidade intelectual. Há muitos casos de abandono escolar. Na medida em que os adolescentes não encontram espaço para a expressão de suas necessidades, podem apresentar comportamentos que são avaliados como inadequados. Os educadores identificam que a resposta para esses comportamentos é a intervenção da psiquiatria e/ou psicologia.

Os sintomas relatados pelas instituições merecem uma escuta criteriosa pois, a maioria diz respeito à não adaptação ao modelo de ensino.

\section{Autonomia eindependência}

O que significa crescer numa instituição, onde há a privação de processos naturais da adolescência, como construir a autonomia, a liberdade e a diferenciação dos outros? Nesse contexto é difícil definir o papel dos serviços de saúde mental, mas é evidente que este não pode se restringir a uma oferta individualizada de cuidado e suporte afetivo.

\section{CONSIDERAÇÕES FINAIS}

A construção dos "perfis-ideiais" colocou em evidência aspectos que devem ser considerados na discussão e formulação de modelos assistenciais capazes de produzirem respostas às necessidades da população. Inspirados no Relatório de Saúde para o Mundo 2001/OMS, identificamos quatro campos para o investimento da atenção para os transtornos mentais: médico, comunitário, familiar e da reabilitação.

De acordo com este relatório, as necessidades de atenção aos transtornos mentais e comportamentais pede uma combinação equilibrada de fatores, que foram nomeados como ingredientes: medicação ou farmacoterapia, psicoterapia e a reabilitação psicossocial. Recomenda ainda que para dosar esses ingredientes não é suficiente apenas o esclarecimento do diagnóstico, pois devemos levar em consideração as necessidades do indivíduo, que mudam com a evolução da doença e as condições de vida do paciente.

A partir da análise das características gerais da população que recorre ao serviço, pudemos discriminar quais as propostas e possibilidades para a construção de um novo modelo, que integre e aprimore às ofertas que o serviço já executa, novas ações definidas a partir da vinculação com as necessidades singulares da clientela.

Um plano efetivo de assistência no campo da saúde mental deve incluir:

- formulação clara de projetos terapêuticos individuais, com avaliação periódica e responsabilização dos profissionais envolvidos;

- participação de usuários e familiares na construção dos projetos terapêuticos;

- ações preventivas e outras que possam assegurar a redução dos fatores de risco;

- melhorar o nível de informação de usuários e familiares;

- garantia de um vínculo duradouro do paciente com o serviço, que propicie a continuidade do cuidado e a responsabilização do serviço e equipe de tratamento;

- promover a integração com outros serviços de saúde;

- promover ações intersetoriais com a escola ou outras instituições que possam oferecer respostas à clientela atendida.

No campo das famílias identificamos as seguintes necessidades:

- possibilitar o reconhecimento por parte das famílias das necessidades próprias de qualquer adolescente e do adolescente em tratamento na instituição; 
- compartilhar os pressupostos teóricos que norteiam o tratamento, tais como os conceitos de desinstitucionalização, cidadania, participação, etc;

- possibilitar que as famílias possam oferecer os cuidados necessários para o adolescente com sofrimento psíquico, inclusive em situações de crise;

- cuidar do sofrimento da família;

- possibilitar que a família possa discutir suas necessidades sociais mais amplas, conjuntamente com outras famílias;

- favorecer a possibilidade de criação espaços associativos. atenção deve:

No campo da reabilitação psicossocial a

- sensibilizar a comunidade escolar para o acolhimento da diversidade;

- garantir a existência de uma instância permanente composta pelos técnicos do CAPS e da Educação a fim de construir novas formas de abordagem para as dificuldades de aprendizagem;

- garantir a satisfação das necessidades de inserção na escola e em projetos de geração de renda, e suas mudanças ao longo do tempo considerando a atenção de longa duração;

- no campo comunitário consideramos pertinente;

- estimular o vínculo dos pacientes com outras instituições: escola, centros esportivos e culturais, grupos de geração de renda, etc, buscando integrar e evitar o isolamento;
- sensibilizar a escola, as instituições e a comunidade buscando promover o enfrentamento da discriminação de que são alvo os adolescentes com transtornos mentais;

- estabelecer parcerias com os gestores e trabalhadores dos abrigos para crianças e adolescentes;

- propiciar a discussão das necessidades econômicas e sociais das famílias;

- estabelecer mecanismo de interação com o Poder Judiciário, especialmente com os juízes responsáveis por definir medidas institucionais para adolescentes com transtornos mentais.

Finalmente consideramos que os profissionais que trabalham com adolescentes com transtornos mentais devem considerar a extrema vulnerabilidade e complexas necessidades dessa população. Contemplar as necessidades dos adolescentes na esfera social, educacional, oferecer cultura, lazer, oportunidades e vínculos afetivos, contribui para a prevenção da instalação de transtornos psíquicos ou atenua a gravidade que estes possam ter.

A trajetória do adolescente para a vida adulta será certamente vivida com períodos de crise e sofrimento, e o sucesso ou o fracasso pessoal, está subordinado ao suporte oferecido por todos os atores que o cercam. Portanto, a atenção em saúde e em saúde mental para os adolescentes, compreende a identificação das necessidades e as a promoção de práticas intersetoriais capazes de desenharem uma política conseqüente destinada a essa população.

MÂNGIA, E. F.; ASSUMPÇÃO, C. N.; QUINTA, J. M.; RUFINO, M. F. Necessities of adolescents with mental disorders. Rev. Ter. Ocup. Univ. São Paulo, v. 14, n. 3, p. 123-32, set./ dez. 2003.

\begin{abstract}
This current study has as finality identify the necessities of adolescents with mental disorders, in the mental health approach, materializing the formulation of more effective actions that can orient the practice of services designated to this population. To reach this purpose, an exploratory research was made, to discuss and link the theorical reference with the "ideal tipes", built hypothetically through the experiences of the team that realized this work. The study of necessities allowed the identification of the actors that must be involved in the answers to the health problems presented, subsiding the analysis and planning the prevent actions and attention in mental health, suggesting alternatives in the field of institutions and health politics area.
\end{abstract}

KEY WORDS: Geriatric psychiatry. Art therapy. 


\section{REFERÊNCIAS}

ALMEIDA, A. O. O problema é de gente grande? Rev. Saúde Paulista da UNIFESP, n. 0, p. 28-1, abr./jun., 2003.

AMARANTE, P. Loucura, cultura e subjetividade: conceitos e estratégias, percursos e atores da reforma psiquiátrica brasileira. In: FLEURY, S. (Org.). Saúde e democracia: a luta do CEBES. São Paulo: Lemos, 1997.

AYRES, J. R. C. M.; FRANÇA JÚNIOR, I. Saúde do adolescente. In: SCHRAIBER, L. B.; NEMES, M. I. B.; MENDES-GONÇALVES, R. B.(Org.). Saúde do adulto. Programas e ações na Unidade Básica. São Paulo: Hucitec, 1996.

BARROS, D. D. Jardins de Abel - desconstrução do manicômio de Trieste. São Paulo: Lemos/EDUSP, 1994.

CECÍLIO, L. C. O. As necessidades de Saúde como conceito estruturante na luta pela integralidade e equidade na atenção em saúde In: CECÍlLIO, L. C. O. Os sentidos da integralidade. Rio de Janeiro: UERJ, IMS, ABRASCO, 2001.

LAURIDSEN, E. P. P. Atenção à saúde mental infantil: um desafio às práticas de saúde. 1998. 135f. Dissertação (mestrado) Faculdade de Saúde Pública da Universidade de São Paulo. São Paulo, 1998.

MANDU, E. N. T.; ALMEIDA, M. C. P. Necessidades em saúde: questões importantes para o trabalho da enfermagem.
Rev. Bras. Enf., Brasília, v. 52, n. 1, 1999.

MINAYO, M. C. S. Pesquisa social teoria, método e criatividade. 7.ed. Petrópolis: Vozes, 1997.

KINOSHITA, R. T. Em busca da cidadania. In: CAMPOS, F. C. B.; HENRIQUES, C. M. P. (Org.). Contra a maré, à beira mar: a experiência do SUS em Santos. São Paulo: Scritta, 1996. p. 39-49.

OMS/OPAS. Relatório sobre a saúde no mundo - 2001. Saúde mental: nova concepção, nova esperança. Gráfica Brasil, Organização Mundial da Saúde, 2001.

SAGGESE, E.;LEITE, L. C. Saúde mental na adolescência Caderno juventude saúde desenvolvimento, v. 1. Brasília, 1999. p.197-205.

SARACENO, B. Libertando identidades - da reabilitação psicossocial à cidadania possível . Belo Horizonte: Instituto Franco Basaglia/Tecorá, 1999.

SCHRAIBER, L. B.; MENDES-GONÇALVES, R. B. Necessidades de saúde e atenção primária. In: SCHRAIBER, L. B.; NEMES, M. I. B.; MENDES-GONÇALVES, R. B. (Org.). Saúde do adulto: programas e ações na unidade básica. São Paulo: Hucitec, 1996.

VASCONCELOS, E.M. Complexidade e pesquisa interdisciplinar. São Paulo: Vozes, 2002. 